\title{
Detection of periods for T Tauri stars ${ }^{\star, \star \star}$
}

\author{
M. Osterloh ${ }^{1}$, E. Thommes ${ }^{2}$ and U. Kania ${ }^{2}$ \\ 1 MPG Research Unit "Staub in Sternentstehungsgebieten", Schillergäßchen 2-3, 07745 Jena, Germany \\ e-mail: osterloh@sol.astro.uni-jena.de \\ 2 MPIA Heidelberg, Königstuhl 17, 69117 Heidelberg, Germany
}

Received August 11, 1995; accepted March 23, 1996

\begin{abstract}
We present the results of $U B r H_{\alpha} z$ monitoring of a sample of $\mathrm{T}$ Tauri stars. The method of differential CCD photometry was applied. The observations span a range of ten days and show that eleven out of twelve sources varied during this time interval. These variations are correlated in all bands. Periods were detected (confidence level $99.99 \%$ ) for eight out of eleven variable stars; one additional period was found at the $99.9 \%$ confidence level. Of those, one (BP Tau) agrees with previously published periods; two, GM Aur and IQ Tau, do not; six are new detections of periodicities. The sample is drawn from the Taurus-Auriga and Cepheus IV star-forming regions.
\end{abstract}

Key words: stars: evolution - stars: pre main-sequence - stars: rotation

\section{Introduction}

$\mathrm{T}$ Tauri stars show periodic or quasi-periodic continuum variability, with amplitudes typically of tenths of a magnitude. The variability is usually attributed to hot or cool spot-like regions in the stellar photosphere that rotate with the star (Richter et al. 1992; Bouvier et al. 1993, 1994; Herbst et al. 1994). That photometric periods indeed reflect stellar rotation can be established by confirming that they remain constant in time. Further, the apparent distinction between classical and weak-line $\mathrm{T}$ Tauri rotational periods (Bouvier et al. 1993) should be put on a broader statistical basis.

To meet these two requirements, we chose a sample of eleven classical and one weak-line $\mathrm{T}$ Tauri star in the Taurus-Auriga dark clouds and Cepheus IV association; three of these were analysed previously. The sample contains only one weak-line T Tauri star (HV Tau) since a large data set of periods already exists for this class of objects (Grankin et al. 1991; Grankin 1991; Grankin 1994).

\section{Observations and data analysis}

The observations were performed on Calar Alto during ten nights from November 19, 1994 to November 30. The

Send offprint requests to: M. Osterloh

*Based on observations made at the German-Spanish Astronomical Center on Calar Alto, Spain

${ }^{\star \star}$ Appendix: photometric data only available in electronic form can be accessed via anonymous ftp cdsarc.u-strasbg.fr or 130.79 .128 .5
$1.23 \mathrm{~m}$ telescope equipped with a tektronix chip (Tek \# 6 in the Calar Alto manual) was used. All sources were observed in the filters $U, B$, Gunn $r$, Gunn $z$ and $H_{\alpha}$. The $U B$ filters are shifted redwards by $\approx 20 \mathrm{~nm}$ relative to the standard Johnson $U B$ filters (see Table 1). The Gunn $z$ filter is not blocked redwards but starting from $\lambda=1.5 \mu \mathrm{m}$. The chip sensitivity curve makes it an effective $(877 / 95)$ filter, with an open end redwards. For the application of the spot blackbody model (see below), the central wavelength was therefore set to $877 \mathrm{~nm}$. An Oke standard, G191B2B, was observed during two photometic nights for approximate absolute brightness calibration. No attempt was made to transform the derived magnitudes to the Johnson system.

Table 1. Central wavelengths and FWHM of the filters used

\begin{tabular}{rrr}
\hline filter & $\lambda[\mathrm{nm}]$ & $\Delta \lambda[\mathrm{nm}]$ \\
\hline$U$ & 370 & 46 \\
$B$ & 457 & 96 \\
Gunn $r$ & 663 & 108 \\
Gunn $z$ & 910 & 90 \\
$H_{\alpha}$ & 655.4 & 16.3 \\
\hline
\end{tabular}

We tried to achieve uniform time spacing between successive measurements for a given $\mathrm{T}$ Tauri star. A typical value was $\approx 30 \mathrm{~min}$. Also, the interval between measurements through the same filter was aimed to be equal, typically $3 \mathrm{hrs}$. Thus, good time coverage for the following harmonic analysis was reached. 
The sample consisted of $\mathrm{T}$ Tauri stars most of which were detected at 1.3 millimeter wavelengths. Quasi-periods had already been detected for three sources; those were included to check if the periods had changed with time. Table 2 lists the sources, together with $H_{\alpha}$ equivalent width, mm-flux density and photospheric excess in $\mathrm{K}$. These quantities suggest the presence of accretion and circumstellar disks.

The weather was clear with the exception of the nights Nov. $20 / 21$, Nov. $21 / 22$, which were partially cloudy. The moon stood close (within $15^{\circ}$ ) to the sources during the first three nights, introducing a strong, non-flat background over the frame, thus increasing the measurement uncertainties.

Differential photometry was performed by comparing the targets to stars in the same field. The field of view amounted to $9 ! 6 \times 9 ! 6$, allowing for one to 13 comparison stars. First, the individual light curves of the stars were plotted on top of each other. Those that seemed to follow a common track (i.e. having parallel line sections) were chosen to construct an average light curve for the T Tauri star: For each of them, the difference in magnitudes to the $\mathrm{T}$ Tauri star was determined at each time point and weighted by its uncertainty. The weighted average over all times gave the magnitudinal difference $d_{j}$ between the $\mathrm{j}^{\text {th }}$ comparison and $\mathrm{T}$ Tauri star.

Then, to obtain the brightness expected for a $\mathrm{T}$ Tauri star at a given time, the weighted average of all field star magnitudes minus the time-averaged differences was computed:

$$
m_{0 i \exp }=\frac{\sum_{j \neq 0} \frac{m_{j i}-d_{j}}{\sigma_{j i}^{2}}}{\sum_{j \neq 0} \frac{1}{\sigma_{j i}^{2}}},
$$

where $j$ is the star index and $i$ is the time index. The T Tauri star is labeled $0 . m$ denotes the magnitude, $\sigma$ the uncertainty.

A photometric variation was considered to be present if the difference between the expected and the measured magnitude exceeded three times the uncertainty estimated from secondary standard measurements or photon statistics. This procedure also gave way for double-checking: Had a field star proved to be variable owing to this criterion, it would have been removed from the list of comparison stars. The uncertainty $\sigma$ follows as the weighted sum of all individual $\sigma \mathrm{s}$ :

$$
\sigma_{0 i}=\left(\sum_{j \neq 0} \frac{1}{\sigma_{j i}^{2}}\right)^{-\frac{1}{2}} .
$$

In three cases (UY Aur, FT Tau, DO Tau), no good comparison stars were available. For those, a $\tau$-model describing the atmospheric extinction was used based on the following procedure: Two secondary standards from the field GM Aur were chosen and an absolute magnitude was determined in the different colors using the good photometric nights. Thus, the two stars (which had color indices encompassing GM Aur's) could be used to monitor the atmospheric extinction $\tau$. The differences of the two $\tau$ 's derived from the blue and red secondary at a given time point amounted to less than the typical scatter of the data, which itself was determined by intercomparison of the different field stars. Thus, color effects in the $\tau$ model should not play any significant role for these stars, therefore neither for GM Aur. Extrapolating to the colors of FT and DO Tau shows these effects to be neglibible for these stars as well.

The arithmetic mean of the two $\tau$ 's was then taken as the typical atmospheric extinction at the measurement time. Cubic spline interpolation (see, e.g., Lancaster \& Salkauskas 1986) was used for a smooth $\tau$-model. The result is shown for the filters Gunn $z$ and $U$ in Figs. 1, 2. The average uncertainty in the filters amounts to $0.05 \mathrm{mag}$ in $U$ and 0.02 in the colors $B$ to Gunn $z$. These are the errors quoted in the photometry for FT Tau, UY Aur and DO Tau (see appendix) if the individual photon statistics do not give rise to higher $\sigma \mathrm{s}$.

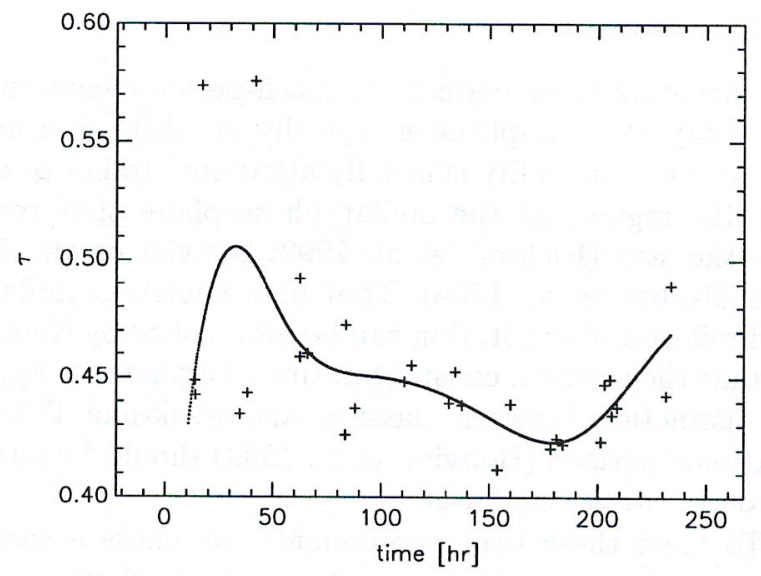

Fig. 1. The fitted $\tau(t)$-function describing the atmospheric extinction in $U$ as a function of time. The time is counted in hours, starting with JD 2449676.0. The crosses are actual data, namely the average of the derived $\tau$ of a blue and a red field star in the GM Aur-field. The solid curve is a cubic spline fit. The average uncertainty in $U$ is $\approx 0.05 \mathrm{mag}$. The fit lies inside of these error bonds, except for the first three nights, were the moon was too close to the field

The resultant differences in magnitudes were mediancentered and subsequently analysed with the technique of the Lomb periodogram (Scargle 1982; Lomb 1976). For this analysis, all data were normalized to common unit variance in each individual filter; the whole set for a given star then provided the input for the analysis. This procedure is justified by the generally good correlation of the data (see below). 
Table 2. Sample stars. The numbers in brackets are the measurement uncertainties. The mm-flux densities are from the surveys of Beckwith et al. (1990) and Osterloh \& Beckwith (1995). The e.w. values are from Herbig \& Bell (1992) (HBC). The color Excesses in K are from Strom et al. (1989)

\begin{tabular}{rlrrr}
\hline HBC No. & Source name & $H_{\alpha}$ e.w. $[\AA]$ & $F_{1.3 \mathrm{~mm}[\mathrm{mJy}](\sigma)}$ & $\Delta \mathrm{K}[\mathrm{mag}]$ \\
\hline 326 & MacC sH15 & 15 & $7(4)$ & \\
4 & MacC H10 & 24 & 11 & \\
5 & MacC H9 & 48 & $10(4)$ & \\
6 & LkHa 200 & 27 & $<14$ & \\
32 & BP Tau & 40 & $37(15)$ & 0.26 \\
384 & FT Tau & & $130(14)$ & \\
41 & IQ Tau & 7.8 & $87(11)$ & 0.32 \\
67 & DO Tau & 100 & $136(11)$ & 0.58 \\
418 & HV Tau & 4.5 & $40(6)$ & \\
75 & DS Tau & 59 & $25(6)$ & \\
76 & UY Aur & 60 & $29(6)$ & 0.48 \\
77 & GM Aur & 96 & $253(12)$ & 0.09 \\
\hline
\end{tabular}

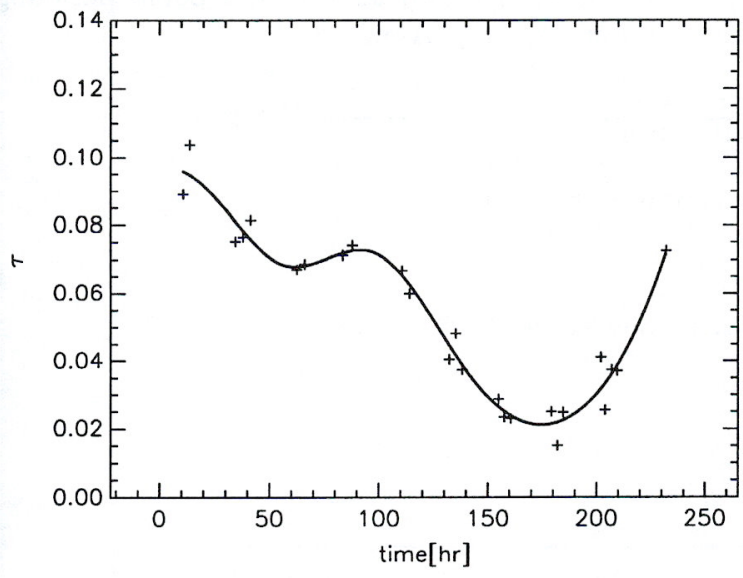

Fig. 2. The fitted $\tau(t)$-function describing the atmospheric extinction in $z$ as a function of time. Symbols and time zeropoint are as in Fig.1

\section{Results and discussion}

\subsection{The data}

The appendix gives the resulting, median-centered differences in magnitude for the variable stars; the $\sigma$-values are the maximum of photon statistics errors (Eq. 2) and the uncertainties estimated from intercomparing any constant field star to the rest of the comparison stars. The time is counted in days, starting with JD 2449676.0. For each star, we give tentative absolute brightness values deduced from Walsh's (1992) spectrophotometric data of G191B2B at our filter center wavelengths and zeroshifted to $\alpha$ Lyr. From least-squares fitting of G191B2B's magnitudeairmass diagram, the uncertainty in the absolute flux calibration is estimated to be $\approx 0.05 \mathrm{mag}$. HV Tau proved to be constant during our observing run; thus, no data are given for it. It is interesting that this is the only weak-line $\mathrm{T}$ Tauri star in our sample.
The lightcurves (except for GM Aur and IQ Tau, see below) are displayed elsewhere (Osterloh 1995), showing already two features: Correlated and sinusoidal variability. In Table 3, we give the correlation coefficients between any two colors of the variable stars. A pair was included for the calculation of a coefficient if the time difference between the two measurements was smallest and not greater than three hours. Changing the maximum time difference to a smaller value does not alter the coefficients significantly. Those are generally high; two exceptions are MacC H9 and the badly correlated $z$ variations of DO Tau. In both cases, a low signal/noise ratio is responsible for this.

\subsection{Harmonic analysis and the spot model}

The Lomb periodograms are shown by Osterloh (1995). The periods derived, with level of significance $\alpha$, are given in Table 4. For comparison, periods from the literature are also quoted in the table. $\alpha$ is derived from MonteCarlo simulations with $10^{4}$ random data sets spaced in time as the original measurements. The uncertainties $\sigma$ result from a $\chi^{2}$-analysis with two free parameters: the period and the amplitude. They refer to a $68.3 \%$ confidence level. The analysis was performed for the whole data set including all colors.

The sinusoidal fits to the lightcurves are good in general, as the small $\alpha$ in Table 4 implies. However, the absolute maximum of IQ Tau's Lomb periodogram occurs at a period of 1.125 days. The $12.5 \mathrm{~d}$-period quoted in Table 4 is only the second highest peak. The two periods can be dependent since the total observing time was ten days. We chose the 12.5 day-period since it is just twice that given by Bouvier et al. (1994). The latter is clearly not present in our data; but a direct inspection of the magnitudes tabulated in Bouvier et al. (1994) shows that two brightness minima in $U$ are separated by 12 days, thus argueing for our period. However, our lightcurve seems to be a combination of a short and a long periodicity. We display IQ Tau's various bands in Fig. 3 . 
Table 3. Correlation coefficients between the variable program stars' colors

\begin{tabular}{|rrrrrrrrrr|l|}
\hline$U B$ & $U r$ & $U H_{\alpha}$ & $U z$ & $B r$ & $B H_{\alpha}$ & $B z$ & $r H_{\alpha}$ & $r z$ & $H_{\alpha} z$ & Star \\
\hline 0.69 & 0.23 & 0.66 & 0.34 & 0.83 & 0.73 & 0.74 & 0.75 & 0.84 & 0.70 & GM Aur \\
0.59 & 0.40 & 0.33 & 0.26 & 0.58 & 0.30 & 0.55 & 0.65 & 0.41 & 0.56 & FT Tau \\
0.86 & 0.76 & 0.68 & 0.53 & 0.88 & 0.80 & 0.75 & 0.76 & 0.75 & 0.34 & IQ Tau \\
0.92 & 0.72 & 0.88 & 0.70 & 0.76 & 0.91 & 0.89 & 0.52 & 0.50 & 0.86 & UY Aur \\
0.45 & 0.54 & 0.42 & 0.27 & 0.75 & 0.94 & 0.53 & 0.75 & 0.72 & 0.61 & DS Tau \\
0.91 & 0.88 & 0.80 & 0.08 & 0.83 & 0.70 & 0.13 & 0.92 & 0.36 & 0.17 & DO Tau \\
0.83 & 0.69 & 0.51 & 0.69 & 0.88 & 0.48 & 0.91 & 0.27 & 0.94 & 0.27 & Bp Tau \\
0.80 & 0.81 & 0.79 & 0.42 & 0.80 & 0.83 & 0.54 & 0.80 & 0.74 & 0.70 & LkHa 200 \\
-0.06 & -0.17 & 0.28 & 0.08 & 0.33 & -0.12 & 0.42 & -0.49 & 0.41 & 0.03 & MacC H9 \\
0.63 & 0.34 & 0.19 & 0.73 & 0.39 & 0.94 & 0.00 & 0.35 & 0.05 & 0.18 & MacC sH15 \\
0.38 & 0.30 & 0.46 & 0.39 & 0.79 & 0.81 & 0.74 & 0.80 & 0.80 & 0.65 & MacC H10 \\
\hline
\end{tabular}

Table 4. Periods detected at Calar Alto for classical T Tauri stars. $\alpha$ is the level of significance in the Lomb periodogram statistics. Formerly detected periods with references are given in the last column for comparison

\begin{tabular}{rrrl} 
Source & Period [days] $(\sigma)$ & $\alpha$ & other Periods \\
\hline BP Tau & $7.7(1.0)$ & $10^{-4}$ & 7.6, Vrba 1986 \\
FT Tau & $8.3(0.1)$ & $10^{-4}$ & \\
IQ Tau & $12.5(2.0)$ & $10^{-4}$ & 6.25, Bouvier et al. 1994 \\
DO Tau & $12.5(1.8)$ & $10^{-4}$ & \\
DS Tau & $7.7(0.6)$ & $10^{-4}$ & \\
UY Aur & $6.7(0.8)$ & $10^{-4}$ & \\
GM Aur & $5.3(0.2)$ & $10^{-4}$ & $11.9,12.0$, Bouvier et al. 1993,1994 \\
LkH 200 & $4.0(0.03)$ & $10^{-4}$ & \\
MacC H9 & $15(2.3)$ & $10^{-3}$ &
\end{tabular}

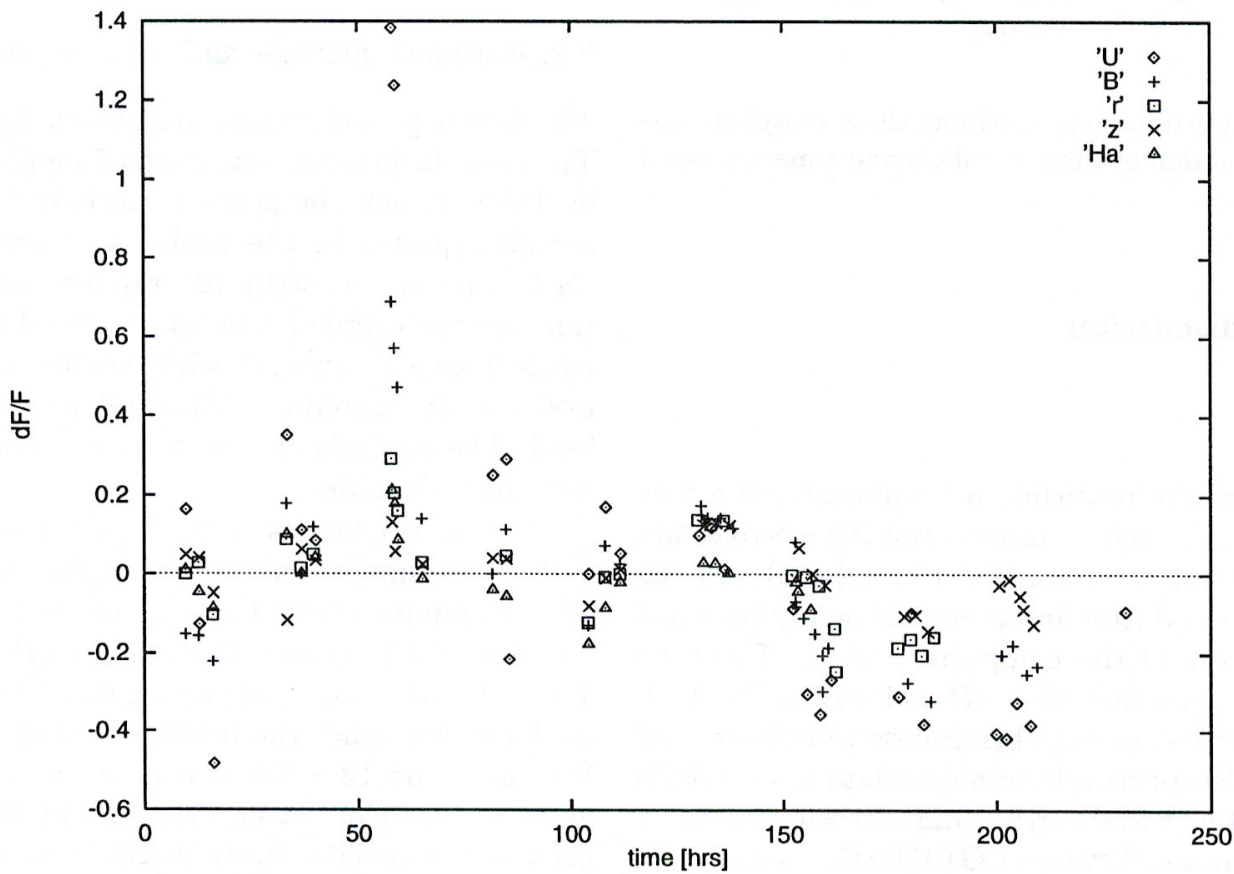

Fig. 3. IQ Tau's lightcurve in the colors $U$ (diamonds), $B$ (upright crosses), $r$ (squares), $H_{\alpha}$ (triangles) and $z$ (crosses). It seems to be a combination of two sinusoidals, one with period of $\approx 3-4$ days, the other with the period quoted in Table 4 . Shown are the relative flux changes, $\mathrm{d} F / F$, not the magnitude differences quoted in the appendix. The time zeropoint is JD 2449676.0 
Table 5. Relative flux ratio amplitudes as a result of Lomb fitting at the different wavelengths. For Gunn $z$, the effective central wavelength $\lambda=877 \mathrm{~nm}$ is quoted

\begin{tabular}{|c|c|c|c|}
\hline Source & $\lambda[\mu]$ & Amplitude $\left(\frac{\Delta F}{F}\right)$ & $\sigma$ \\
\hline \multirow[t]{5}{*}{ GM Aur } & 0.370 & 0.171 & 0.090 \\
\hline & 0.460 & 0.101 & 0.037 \\
\hline & 0.660 & 0.079 & 0.009 \\
\hline & 0.877 & 0.055 & 0.026 \\
\hline & 0.656 & 0.111 & 0.006 \\
\hline \multirow{5}{*}{ FT Tau } & 0.370 & 0.195 & 0.060 \\
\hline & 0.460 & 0.124 & 0.100 \\
\hline & 0.660 & 0.110 & 0.030 \\
\hline & 0.877 & 0.063 & 0.025 \\
\hline & 0.656 & 0.169 & 0.030 \\
\hline \multirow[t]{5}{*}{ IQ Tau } & 0.370 & 0.372 & 0.150 \\
\hline & 0.460 & 0.247 & 0.100 \\
\hline & 0.660 & 0.131 & 0.050 \\
\hline & 0.877 & 0.065 & 0.035 \\
\hline & 0.656 & 0.031 & 0.030 \\
\hline \multirow[t]{5}{*}{ UY Aur } & 0.370 & 0.530 & 0.100 \\
\hline & 0.460 & 0.384 & 0.140 \\
\hline & 0.660 & 0.243 & 0.110 \\
\hline & 0.877 & 0.121 & 0.080 \\
\hline & 0.656 & 0.215 & 0.100 \\
\hline \multirow[t]{5}{*}{ DS Tau } & 0.370 & 0.514 & 0.350 \\
\hline & 0.460 & 0.257 & 0.060 \\
\hline & 0.660 & 0.423 & 0.120 \\
\hline & 0.877 & 0.342 & 0.135 \\
\hline & 0.656 & 0.289 & 0.030 \\
\hline \multirow[t]{5}{*}{ DO Tau } & 0.370 & 0.360 & 0.040 \\
\hline & 0.460 & 0.365 & 0.125 \\
\hline & 0.660 & 0.261 & 0.050 \\
\hline & 0.877 & 0.051 & 0.018 \\
\hline & 0.656 & 0.240 & 0.130 \\
\hline \multirow[t]{5}{*}{ BP Tau } & 0.370 & 0.286 & 0.145 \\
\hline & 0.460 & 0.193 & 0.045 \\
\hline & 0.660 & 0.180 & 0.050 \\
\hline & 0.877 & 0.107 & 0.017 \\
\hline & 0.656 & 0.183 & 0.050 \\
\hline \multirow[t]{5}{*}{$\mathrm{LkH} \alpha 200$} & 0.370 & 0.207 & 0.080 \\
\hline & 0.460 & 0.097 & 0.040 \\
\hline & 0.660 & 0.067 & 0.028 \\
\hline & 0.877 & 0.041 & 0.025 \\
\hline & 0.656 & 0.077 & 0.030 \\
\hline \multirow{5}{*}{$\mathrm{Mac}$ CH9 } & 0.370 & 0.073 & 0.199 \\
\hline & 0.460 & 0.080 & 0.037 \\
\hline & 0.660 & 0.028 & 0.043 \\
\hline & 0.877 & 0.030 & 0.027 \\
\hline & 0.656 & 0.003 & 0.034 \\
\hline
\end{tabular}

We give the Lomb amplitudes of the sinusoidals in Table 5 . They are plotted in Fig. 4 . The $\sigma$ s in Table 5 result from a $\chi^{2}$-analysis with two degrees of freedom (frequency and amplitude) and the requirement that the level of confidence be $68.3 \%$. The lack of plateaus in the light curves, their apparent sinusoidal shape as well as the fact that we find, within statistical uncertainties, as many brightness values above as below their arithmetic mean, makes us choose a special case of Eq. (1) in Bouvier et al. (1993) to fit the amplitudes to a two-component blackbody model:

$$
2 \frac{\Delta F_{\nu}}{F_{\nu}}=f \frac{B_{\nu}\left(T_{2}\right)-B_{\nu}\left(T_{1}\right)}{B_{\nu}\left(T_{1}\right)} .
$$

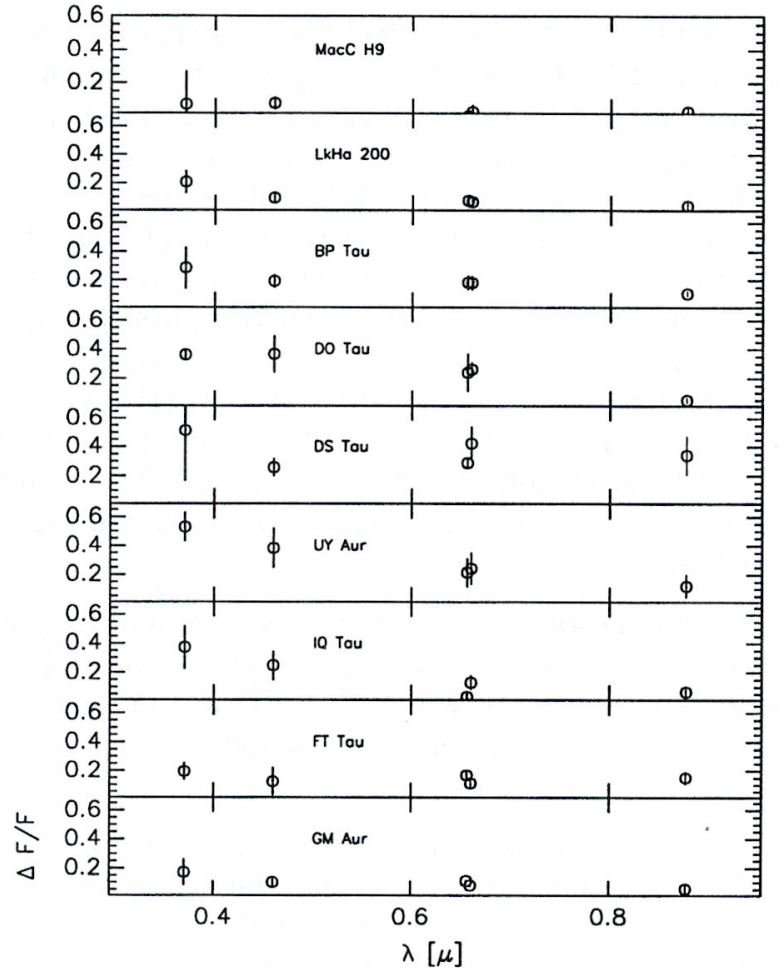

Fig. 4. Relative flux ratio amplitudes as a result of Lomb fitting at the different wavelengths. Note that we have used the effective central wavelength $877 \mathrm{~nm}$ of Gunn $z$, not the central filter wavelength (see text)

Here, $\Delta F_{\nu}$ is the Lomb amplitude of the variation of the flux density $F_{\nu}$, at frequency $\nu ; f$ is the maximum fraction of the visible stellar disk that is covered by the spot, and $B_{\nu}(T)$ is the Planck blackbody function at frequency $\nu$ and temperature $T$, with index 1,2 referring to star and spot, respectively. In Bouvier's Eq. (1), the fraction $f_{2}$ has been set to zero, due to the reasons outlined above. Also, limb darkening effects have been neglected in the equation; we estimate the effects of this simplification as follows:

With limb darkening included, Eq. (3) contains the additional factor

$$
\frac{1-\mu+\mu \cos \alpha}{1-\frac{\mu}{3}}
$$

where $\mu$ is the wavelength-dependent coefficient of limb darkening, and $\alpha$ is the angle between the spot normal at maximum projected surface, and the line of sight (Torrez \& Ferraz Mello 1973). Using solar values for $\mu$ found in Pierce \& Slaughter (1977), we find that the strongest deviations in $U$ amount to an increase of the amplitude by $\approx 42 \%$; this occurs when the spot is at low latitudes with respect to the line of sight $(\cos \alpha \rightarrow 1)$. This increase is about two times the typical uncertainties in our amplitude determinations (cf. Table 5). From Eq. 
(4), it can be seen that the effect becomes smaller when going to higher latitudes. The average effect, for small spots confined mainly to one latitude, is an increase by $15 \%$ of the amplitude, leading to a systematic overestimate of the effective covered area by $15 \%$. This overestimate is reduced to $8 \%$ when going to wavelengths of $7000 \AA$. At high latitudes $(\cos \alpha \rightarrow 0)$, the amplitudes are reduced to $14 \%$. However, the contribution to the projected amplitude drops with $\cos \alpha$; thus, when a fractional coverage of the spots by $12 \%$ is derived from Eq. (3) (this being the minimum of $f$ in Table 6 below), the spots must cover the star at least down to latitudes of $40^{\circ}$. The resulting underestimate of $f$ now only amounts to $9 \%$ in $U$, and $5 \%$ at $7000 \AA$.

A combination of a continuum source and a spotted photosphere (continuum veiling) produces an underestimate of the real amplitudes, thus of the spot fractional coverage $f$. If the continuum is colored, the derived spot temperature will change.

Below we will show that the uncertainties in the amplitude determination lead to a large region of allowed temperature-f-combinations. It therefore does not seem adequate to derive more than gross properties from the deduced amplitudes. Whenever the simplest spot model, Eq. (3), fails, we will try to reconcile the picture by invoking effects of veiling and limb darkening. For now, let us proceed with Eq. (3).

$T_{1}$ follows from the spectral type found in Herbig \& Bell (1992) (HBC); the corresponding effective temperature was taken from Cohen \& Kuhi 1979. We do a $\chi^{2}$-fit of Eq. (3) to the UBrz amplitudes and estimate the uncertainties $\sigma$ by inspecting the $\chi^{2}+2.3$ - line (two degrees of freedom). This line is replaced whenever $f$ becomes larger than 1.0: Eq. (3) $\left(f_{2}=0\right)$ cannot be assumed to be valid any longer then; for a three-parameter-fit ( $f_{2}$ included), it is not likely that 4 amplitudes will give reliable results. Therefore, we quote only our 2-parameter-fits in Table 6, for the sources where $\chi^{2}$ is less than 2 . If the upper limit $f=1$ implies a limit in the spot temperature, an entry "UL" is found in Col. 6 of the table. If $f=1$ is already the best fit of the amplitudes, only lower limits for $f$ and corresponding temperature ranges are given. The case $f=1$ is, of course, only to be understood to be the best mathematical fit; physically, it is very unlikely that such a huge "spot" would still allow Eq. (3), to be applied with $f_{2}=0$.

Usually, the amplitude uncertainties imply spot temperature uncertainties of several hundreds of Kelvin, allowing for an even wider span of $f$-values. The $\chi^{2}+2.3-$ criterion proves to be weak and allows for spots with $f>1.0$ in many cases. GM Aur and BP Tau can be fitted equally well with a hot or a cool spot. Only for two cases, IQ Tau and UY Aur, a $\chi^{2}$ less then two is found for only one type of spot; this is a hot spot for both sources. To unambigously determine the spot parameters, it would therefore be desirable to obtain a larger stock of data either by spectro-photometry or with more filters at hand.

For the sources DS Tau and DO Tau, no good fit could be achieved. The uncertainties for $\mathrm{MacC} \mathrm{H} 9$ are so high that any temperature- $f$ combination is allowed. We now want to discuss the two former sources in more detail.

\subsection{The spot model: Badly fitted sources}

DO Tau has about equal $U$ and $B$ amplitudes $\left(\frac{\Delta F}{F} \approx 0.36\right)$ implying a spot temperature which is lower than the photospheric one (about $3000 \mathrm{~K}$ ). This, in turn, requires a Gunn $z$-amplitude higher than the one being measured $\left(\frac{\Delta F}{F} \approx 0.28\right.$ as compared to the measured $\left.\frac{\Delta F}{F} \approx 0.05\right)$. Limb darkening color effects produce a maximum of $20 \%$ underestimate in Gunn $z$ (using Eq. (4) with $\cos \alpha=1$ and coefficients of Pierce \& Slaughter 1977) and thus cannot explain our findings.

A possible explanation can be found by noting that the $H_{\alpha}$-amplitudes equal those found in Gunn $r$; the high equivalent width (e.w.) of DO Tau (100 $\AA$, HBC) suggests that about $50 \%$ of the total flux measured in Gunn $r$ originates from the $H_{\alpha}$-line. Neither line- nor continuumconfined variability can therefore explain the equal amplitudes; both would result in about half the broadband relative variation as compared to the line filter. If we assume that equal percentages of the periodic variations were due to lines and continuum, respectively, we would have an explanation for the low Gunn $z$-amplitude, where emission lines as a source of additional variability are missing.

An alternative explanation would be an additional continuum source emerging in the red, possibly a disk. Strong mm-emission was found for the source by Beckwith et al. (1990), indicating outer disk material. Strom et al. (1989) note a strong color excess in $\mathrm{K}$ due to, in this interpretation, inner disk material. Up to now, no infrared companion was detected for DO Tau which could account for this excess (Leinert et al. 1993). Beckwith et al. (1990) estimated the DO Tau disk parameters; these allow for only $4 \%$ disk contribution to the total $z$-band emission. "Disk veiling" (for a definition, see Basri \& Batalha 1990) must be $\approx 1.3$ in Gunn $z$ and 0 in Gunn $r$ to account for the lowest $r / z$ amplitude ratio within the $1 \sigma$-uncertainties. Thus, we must have a non-standard disk (very hot in the inner parts) for this alternative. It is likely that, for the inner parts, the thin disk approximation would have to be abandoned to enable a more effective heating by the central star.

$D S$ Tau. Formally, DS Tau can be fitted with a spot of temperature $T_{2} \leq 2300 \mathrm{~K}$ covering $62 \%$ of the stellar disk. But the $\chi^{2}$ is much poorer (2.1) than in the other blackbody fits. The reason is a Gunn $z$ amplitude too high for the spot model, as compared to the $B$ amplitude. The effect cannot be explained by limb-darkening, since such a large spot would extend down to latitudes of $10^{\circ}$, giving an effective underestimate of Gunn $z$ relative to $U$. Basri \& 
Table 6. Two-component blackbody model fit to the sources with $\chi^{2}<2.0$; the symbols are defined in the text

\begin{tabular}{rrrrrrr}
\hline Name & $T_{1}[\mathrm{~K}]$ & $T_{2}[\mathrm{~K}]$ & $\sigma[\mathrm{K}]$ & $f$ & $\sigma$ & $\chi^{2}$ \\
\hline GM Aur & 4000 & 4100 & +600 & 1.0 & -0.86 & 0.2 \\
& 4000 & 3900 & -1400 & 1.0 & -0.82 & 0.3 \\
FT Tau & 3890 & 4100 & +700 & 0.62 & -.52 & 0.4 \\
& 3890 & 3700 & -800 & 0.78 & -.56 & 0.9 \\
IQ Tau & 3800 & 4600 & $+700-600$ & 0.14 & UL $1.0,-0.08$ & 0.3 \\
UY Aur & 4000 & 4650 & $+1000-350$ & 0.34 & UL $1.0,-0.28$ & 0.2 \\
BP Tau & 4000 & 4200 & +600 & 1.0 & -0.82 & 0.9 \\
& 4000 & 3800 & -700 & 1.0 & -0.66 & 0.8 \\
LkH $_{\alpha} 200$ & 5100 & 6300 & $+900-1000$ & 0.10 & UL $1.0-0.04$ & 0.5 \\
& 5100 & 4900 & $+50-1600$ & 0.80 & UL $1.0-0.66$ & 1.7 \\
\hline
\end{tabular}

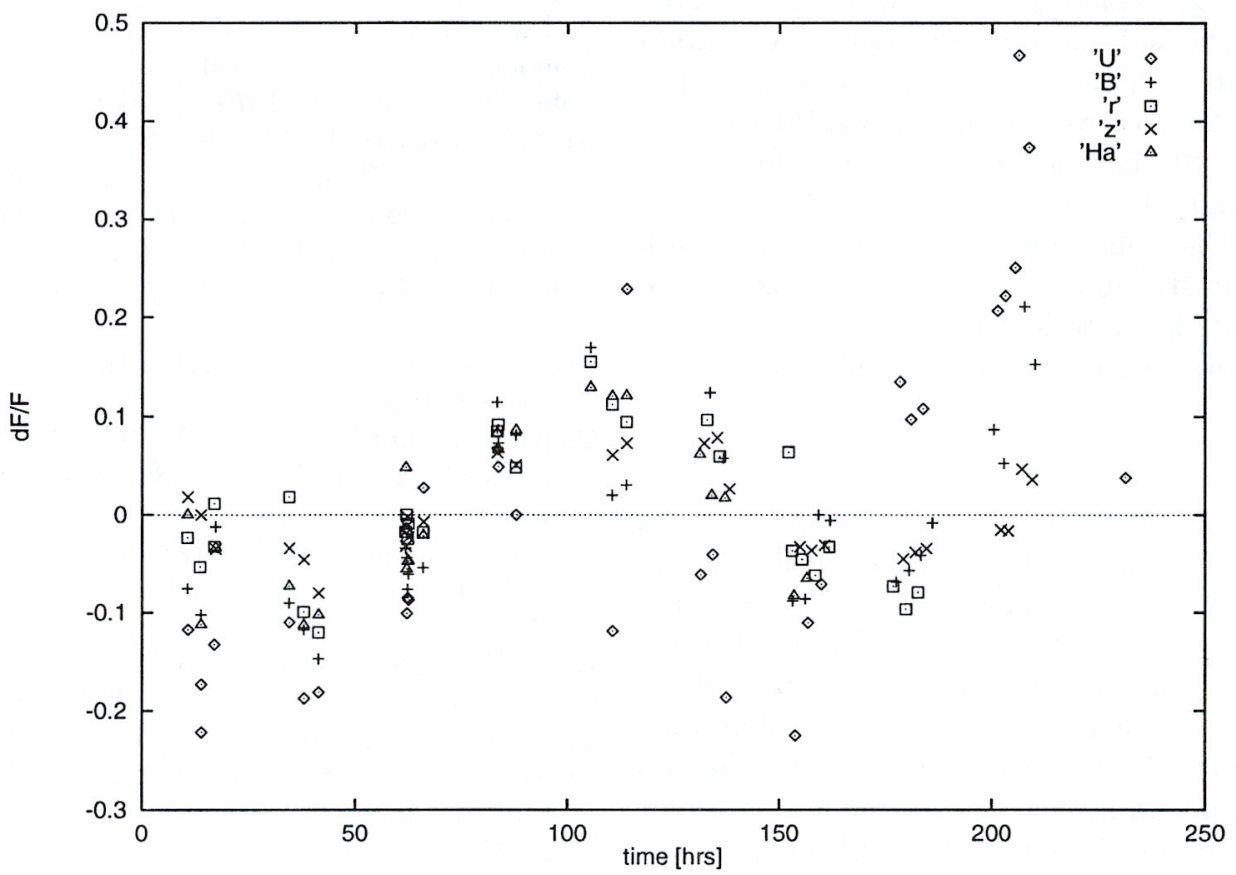

Fig. 5. GM Aur's lightcurve in the colors $U, B, r, H_{\alpha}$ and $z$. Color code and time zeropoint are as in Fig. 3. No indication of a $12 \mathrm{~d}$ period with Bouvier et al. (1994) amplitudes is present. Instead, a sinusoidal variation with the 5.3d-period quoted in Table 4 can be recognised

Batalha (1990) only note a moderate veiling (0.5) at $\mathrm{H} \beta$. A strong decrease of this veiling towards the red would be required to yield a spot-like behavior of the amplitudes, but would be possible.

Does additional reprocessing by disks in the red provide an alternative explanation to blue veiling? DS Tau has medium K excess (0.23, Strom et al. 1989); also, the far IR flux densities suggest a disk with a temperature profile close to the one expected for standard flat accretion disks (Beckwith et al. 1990). It is thus not likely that disk variation contributes significantly in Gunn $z$.

A third alternative is that the derived "spot" temperature is real. The low amplitude in $H_{\alpha}$ as compared to Gunn $r$ plus the medium e.w. (59 $\AA$ ) imply that the variation is consistent with the blackbody model. No IR companion was detected by Leinert et al. (1993). Either there are very cool spots on the surface, or large or many occulting bodies are responsible for the periodic variation. If the observed period corresponds to their orbital motion, the resulting distance (with DS Tau's mass of $1.3 \mathrm{M}_{\odot}$ ) corresponds to 7.5 star radii, or $0.08 \mathrm{AU}$.

\subsection{The spot model: Alternating periods}

Two out of three periods disagree with those found in the literature: IQ Tau (see above) and GM Aur. For IQ Tau, Bouvier et al. data show lightcurve peaks in $U$ separated by a time corresponding to our period. This feature is not as eminent but probably present in the other colors. Bouvier et al. data can be described by a sine-wave of our frequency, together with a general decrease in brightness. It is interesting that our data set exhibits the same trend. 
Yet, our data additionally show a superimposed shorter sine-wave, with a period of $\approx 3-4$ days. This superposition of at least three periods (zero frequency, main and superposed shorter frequency) complicates the analysis. It is possible that alternating, as well as higher periods occur due to variable circumstellar extinction.

GM Aur's case is particularly interesting since it was Lomb-analysed twice by Bouvier $(1993,1994)$ and still gave the same period disagreeing with the one found by us. Clearly, we are not able to deny a 12d-period with observations spanning 10 days, but the periodogram shows no maximum at all at the corresponding frequency. Also, the direct lightcurve (Fig. 5) clearly favours our period. However, our amplitudes and those in Bouvier et al. (1994) are remarkably similar (note that we refer to sine amplitudes in Table 5; the total variation is twice that value). No companion nor spot can make up for the difference in periods; also, the lightcurve is clearly sinusoidal with the period derived by the Lomb analysis. We therefore think that variable circumstellar extinction is more likely; the extinguishing clouds must reside in preferred (keplerian) orbits in order to produce observable periodicities.

\section{Conclusions}

Relative photometry is presented for eleven variable sources drawn from a sample of eleven classical and one weak-line T Tauri star. Quasiperiodic variability was detected in nine of them; the confidence level for eight sources was greater than $99.99 \%$. The periods are usually unique, with the exception of IQ Tau whose lightcurve seems to be a superposition of two sines. The amplitudes of the variations argue in favor of a spot model except for the cases DS Tau, where the Gunn $z$ amplitude is abnormally large, and DO Tau, where it is abnormally small. The former case might be explained by the presence of one or several cool companions undetected up to now, or by veiling confined to the blue spectral region. A very hot inner disk, gas ring or companion can be responsible for DO Tau's behavior. A third case where the spot model does not seem to apply is GM Aur, where a period differing from Bouvier et al. (1993, 1994) was found. Variable circumstellar extinction is advocated as an explanation.
Acknowledgements. The observations would have been impossible without the support of the Calar Alto staff, in particular Ulrich Thiele. M.O. thanks Steven Beckwith for valuable suggestions.

\section{References}

Basri G., Batalha C., 1990, ApJ 363, 654

Beckwith S.V.W., Sargent A., Chini R., Güsten R., 1990, AJ 99,924

Bouvier J., Covino E., Kovo O., Martín E.L., Matthews J.M., Terranegra L., Beck S.C., 1994, Université Joseph Fourier, preprint No. 201

Bouvier J., Cabrit S., Fernández M., Martín E.L., Matthews J.M., 1993, A\&A 272, 176

Bouvier J., 1990, AJ 99, 946

Cohen M., Kuhi L.V., 1979, ApJS 41, 743

Grankin K.N., 1991, IBVS No. 3823

Grankin K.N., Ibragimov M.A., Melkinov S.Ju., Shevchenko V.S., Yakubov S.D., 1991, IBVS No. 3658

Grankin K.N., 1994, IBVS No. 4042

Herbig G.H., Bell K.R., 1992, Lick Observation Bulletin 1111 (HBC)

Herbst W., Herbst D.K., Grossman E.J., Weinstein D., 1994, AJ 108, 1906

Cohen M., Kuhi L.V., 1979, ApJS 41, 743

Lancaster P., Salkauskas K., 1986, "Curve and Surface fitting". academic press, p. 94

Leinert Ch., Zinnecker H., Weitzel N., Christou J., Ridgway S.T., Jameson R., Haas M., Lenzen R., 1993, A\&A 278, 129

Lomb N.R., 1976, A\&AS 39, 447

Osterloh M., 1995, Ph.D. Thesis, Heidelberg

Osterloh M., Beckwith S.V.W., 1995, ApJ 439, 288

Pierce A.K., Slaughter C.D., 1977, Sol. Phys. 51, 25

Richter M., Basri G., Perlmutter S., Pennypacker C., 1992, PASP 104, 1144

Scargle J.D., 1982, ApJ 263, 835

Strom K.M., Strom S.E., Edwards S., Cabrit S., Skrutskie M.F., 1989, AJ 97, 1451

Torres C.A.O., Ferraz Mello S., 1973, A\&A 27, 231

Vrba F.J., Rydgren A.E., Chugainov P.F., Shakovskaya N.I., Zak D.S., 1986, ApJ 306, 199

Walsh J.R., 1992, ESO-document 